\title{
Deterioro cognoscitivo en pacientes con tuberculosis del sistema nervioso central
}

\author{
Ignacio Popoca-Rodríguez y Graciela Cárdenas* \\ Instituto Nacional de Neurología y Neurocirugía "Manuel Velasco Suarez", Departamento de Neuroinfectología, Unidad de Investigación Clínica, \\ Ciudad de México, México
}

\section{Resumen}

Introducción: La tuberculosis en México sigue siendo causa importante de morbimortalidad; en los últimos cuatro años, se reportaron 110681 casos de tuberculosis pulmonar y 1571 casos de tuberculosis meníngea. Objetivo: Determinar las secuelas neurocognoscitivas, presentación clínica y alteraciones en los estudios de neuroimagen en pacientes con tuberculosis del sistema nervioso central. Métodos: Se realizó un estudio retrospectivo, analítico y transversal de 2010 a 2019. Se incluyeron pacientes con tuberculosis del sistema nervioso central, con y sin coinfección por VIH/sida. Resultados: Durante el periodo de estudio se incluyeron 104 casos con diagnóstico definitivo y probable de tuberculosis del sistema nervioso central; de acuerdo con los criterios de Marais, $38 \%$ presentó coinfección por VIH/sida y $55 \%$, diversas comorbilidades $(p=0.0001) ; 49 \%$ presentó alteraciones cognoscitivas y 14 \% falleció. Conclusiones: Aunque la infección por VIH/sida puede contribuir al deterioro cognitivo del paciente con tuberculosis meníngea, no se observaron diferencias entre pacientes con y sin VIH/sida. Las secuelas cognoscitivas mostraron mejoría en el seguimiento con el adecuado manejo y control terapéutico de los pacientes.

PALABRAS CLAVE: Deterioro cognitivo. Secuelas cognitivas. Tuberculosis meníngea. Sistema nervioso central. VIH/sida.

\section{Cognitive impairment in patients with central nervous system tuberculosis}

\begin{abstract}
Introduction: Tuberculosis in Mexico remains an important cause of morbidity and mortality; in the last four years, 110,681 cases of pulmonary tuberculosis and 1,571 cases of tuberculous meningitis were reported. Objective: To determine the neurocognitive sequelae, clinical presentation and neuroimaging alterations in patients with central nervous system tuberculosis. Methods: A retrospective, analytical, cross-sectional study was carried out from 2010 to 2019. Patients with central nervous system tuberculosis, with and without HIV/AIDS coinfection, were included. Results: During the study period, 104 cases with a definitive or probable central nervous system tuberculosis diagnosis were included; according to the Marais criteria, $38 \%$ had HIV/AIDS coinfection, and $55 \%$, various comorbidities ( $p=0.0001$ ); $49 \%$ had cognitive alterations, and $14 \%$ died. Conclusions: Although HIV/AIDS infection can contribute to cognitive decline in patients with tuberculous meningitis, no differences were observed between patients with and without HIV/AIDS. Cognitive sequelae showed improvement during follow-up with adequate management and therapeutic control of the patients.
\end{abstract}

KEY WORDS: Cognitive impairment. Cognitive sequelae. Tuberculous meningitis. Central nervous system. HIV/AIDS.

Correspondencia:

*Graciela Cárdenas

E-mail: gracielacardenas@yahoo.com.mx

0016-3813/@ 2021 Academia Nacional de Medicina de México, A.C. Publicado por Permanyer. Este es un artículo open access bajo la licencia CC BY-NC-ND (http://creativecommons.org/licenses/by-nc-nd/4.0/).
Fecha de recepción: 16-10-2020

Fecha de aceptación: 08-03-2021
Gac Med Mex. 2021;157:385-390

Disponible en PubMed

Disponico.com 


\section{Introducción}

La tuberculosis (TB) hoy día es un importante problema de salud en el mundo debido a su alta mortalidad. Es una de las 10 principales causas de muerte y la primera por un solo agente infeccioso, con lo que supera a la infección por $\mathrm{VIH} /$ sida. En este contexto, durante el año 2018 se estimó que en el mundo existieron 10 millones de personas afectadas por TB, la cual ocasionó 1.2 millones de muertes en personas sin infección por $\mathrm{VIH}$ y cerca de 251000 en personas con $\mathrm{VIH} /$ sida. $^{1}$ La TB del sistema nervioso central, particularmente la afección meníngea, es la más grave y potencialmente mortal de todas las formas de afección extrapulmonar. ${ }^{2}$ En los pacientes supervivientes, las secuelas neurológicas son diversas, incluidas las afecciones motoras, sensitivas, hidrocefalia y deterioro cognoscitivo, ${ }^{3-5}$ el cual incluye la pérdida gradual y progresiva de funciones mentales en los dominios de memoria, orientación, abstracción, lenguaje, razonamiento, funciones ejecutivas $y$ habilidades de aprendizaje y visuoespaciales. ${ }^{6,7}$ Aunque es una entidad multicausal, las infecciones sistémicas y del sistema nervioso central parecen tener un papel importante, incluyendo la infección por $\mathrm{VIH} / \mathrm{sida}^{8,9}$

En México, no existe información sobre las secuelas neurológicas en pacientes con TB meníngea, ${ }^{10}$ particularmente el deterioro cognitivo, por ello decidimos realizar un estudio retrospectivo, analítico y transversal de nueve años en pacientes admitidos en un centro de referencia neurológico, para evaluar el compromiso de funciones mentales y deterioro cognoscitivo al momento del egreso hospitalario (afección temprana) y a los 12 meses de seguimiento clínico (largo plazo); de igual forma, se evaluaron los posibles factores de riesgo de dicha población.

\section{Métodos}

Se realizó un estudio retrospectivo, analítico y transversal en pacientes adultos atendidos en el Instituto Nacional de Neurología y Neurocirugía de 2010 a 2019. Se utilizaron los criterios del Consenso Diagnóstico de Tubeculosis Meníngea de Marais et al::11 únicamente se incluyeron pacientes $\geq 15$ años con diagnóstico definitivo y aquellos con diagnóstico probable y respuesta clínico-radiológica a tratamiento empírico con antifímicos.

Durante la hospitalización de los pacientes se les aplicó la prueba Montreal Cognitive Assessment (MoCA) y pruebas neuropsicológicas (NEUROPSI), para evaluación de la memoria a corto plazo, habilidades visuoespaciales, funciones ejecutivas, atención, concentración y memoria de trabajo, lenguaje y orientación temporoespacial.

Todas las variables cualitativas fueron expresadas en porcentajes y su comparación se realizó mediante prueba de $\chi^{2}$. Se consideraron diferencias estadísticamente significativas aquellas con $p<0.05$. Del mismo modo, las variables cuantitativas se expresaron en media \pm desviación estándar. Para las comparaciones se utilizó prueba $t$ de Student $o \mathrm{U}$ de Mann-Whitney, según el comportamiento con la prueba de Kolmogorov-Smirnov. Todos los datos fueron analizados con el programa SPSS versión 22.

La investigación fue aprobada por el Comité de Ética de investigación del Instituto Nacional de Neurología con el registro 96/17.

\section{Resultados}

Durante el periodo de estudio se detectaron 118 pacientes con cuadro clínico sugestivo de TB, quienes fueron atendidos en el Instituto Nacional de Neurología y Neurocirugía, pero solo 104 cumplieron con los criterios de ingreso.

\section{Características epidemiológicas de la población}

Se incluyeron 39 pacientes (38\%) con TB meníngea y coinfección por $\mathrm{VIH} /$ sida y $65(63 \%)$ sin coinfección por $\mathrm{VIH}$. En la Tabla 1 se resumen las características generales de los grupos. Los pacientes del grupo con $\mathrm{VIH} /$ sida fueron significativamente de menor edad $(p=0.04)$. La mediana de la carga viral de VIH (al diagnóstico de la infección por VIH) fue 1111944 (rango intercuartilar [RIC] = 476 822), mientras que la mediana de la carga viral de $\mathrm{VIH}$ (al diagnóstico de TB) fue 69055 (RIC = 196 749). En relación con las subpoblaciones linfocitarias, la mediana de linfocitos T CD4+ (al diagnóstico de VIH) fue $111 / \mathrm{mm}^{3}$ (RIC = 198), mientras que al momento del diagnóstico de TB fue $126 / \mathrm{mm}^{3}(\mathrm{RIC}=208)$.

De acuerdo con los criterios de Marais et al., ${ }^{11}$ en nuestra población se observó diagnóstico definitivo en $30 \%$ y probable en el resto, aunque con adecuada respuesta clínica y radiológica a los antifímicos.

\section{Características clínicas}

En la población global, la presentación clínica más frecuente fue la de meningitis $(62 \%, n=64)$ y la 
Tabla 1. Características generales de los grupos

\begin{tabular}{|c|c|c|c|c|c|}
\hline \multirow[t]{2}{*}{ Característica } & \multicolumn{4}{|c|}{ TB meníngea } & \multirow[t]{2}{*}{$p$} \\
\hline & \multicolumn{2}{|c|}{$\begin{array}{c}\text { Con } \\
\text { coinfección } \\
\text { VIH/sida } \\
(n=39)\end{array}$} & \multicolumn{2}{|c|}{$\begin{array}{c}\text { Sin } \\
\text { coinfección } \\
\text { VIH } \\
(\mathrm{n}=65)\end{array}$} & \\
\hline \multirow{2}{*}{$\begin{array}{l}\text { Edad en años, } \\
\text { mediana (RIC) }\end{array}$} & \multicolumn{2}{|c|}{$31(10)$} & \multicolumn{2}{|c|}{$40(27)$} & \multirow[t]{2}{*}{0.04} \\
\hline & $n$ & $\%$ & $n$ & $\%$ & \\
\hline $\begin{array}{l}\text { Sexo } \\
\text { Masculino } \\
\text { Femenino }\end{array}$ & $\begin{array}{c}37 \\
2\end{array}$ & $\begin{array}{c}95 \\
5\end{array}$ & $\begin{array}{l}35 \\
30\end{array}$ & $\begin{array}{l}54 \\
46\end{array}$ & 0.0001 \\
\hline $\begin{array}{l}\text { Procedencia } \\
\text { Ciudad de México/área } \\
\text { metropolitana } \\
\text { Otro estado }\end{array}$ & $\begin{array}{l}24 \\
15\end{array}$ & $\begin{array}{l}62 \\
38\end{array}$ & $\begin{array}{l}27 \\
38\end{array}$ & $\begin{array}{l}42 \\
58\end{array}$ & 0.06 \\
\hline $\begin{array}{l}\text { Antecedente de TB } \\
\text { pulmonar }\end{array}$ & 6 & 2 & 8 & 1 & 0.7 \\
\hline Comorbilidades & 39 & 68 & 18 & 32 & 0.0001 \\
\hline $\begin{array}{l}\text { Cuadro clínico } \\
\text { Meningitis } \\
\text { Hidrocefalia } \\
\text { Otro }\end{array}$ & $\begin{array}{c}20 \\
2 \\
17\end{array}$ & $\begin{array}{c}51 \\
5 \\
44\end{array}$ & $\begin{array}{c}44 \\
3 \\
18\end{array}$ & $\begin{array}{c}68 \\
5 \\
28\end{array}$ & 0.09 \\
\hline $\begin{array}{l}\text { Desenlace clínico } \\
\text { Supervivencia } \\
\text { Muerte }\end{array}$ & $\begin{array}{c}32 \\
7\end{array}$ & $\begin{array}{l}82 \\
18\end{array}$ & $\begin{array}{c}57 \\
7\end{array}$ & $\begin{array}{l}89 \\
11\end{array}$ & 0.0001 \\
\hline
\end{tabular}

hidrocefalia $(5 \%, n=5)$. Otras manifestaciones (deterioro cognoscitivo, vasculitis secundaria, etcétera) se observaron en $34 \%(n=35)$. No hubo diferencias estadísticamente significativas en la presentación clínica entre pacientes con y sin coinfección por $\mathrm{VIH} /$ sida. El estado de consciencia, evaluado mediante la escala de coma de Glasgow (ECG), presentó una puntuación promedio de $14 \pm 2.2$ al ingreso en toda la población, mientras que al egreso fue $13.3 \pm 4.4$.

\section{Características de neuroimagen}

En todos los pacientes se realizó resonancia magnética (RM), los principales hallazgos fueron meningitis o realce leptomeníngeo $(n=31,24 \%)$, abscesos o colecciones ( $n=38,29 \%$ ), isquemia/infartos $(n=6$, $4.6 \%$ ) e hidrocefalia $(n=5,5 \%)$. Se reportó vasculitis secundaria en 33 pacientes $(25 \%)$ mediante estudio de Doppler transcraneal.

\section{Estudios complementarios}

En todos los casos se procesaron cultivos bacteriológicos en medio Lowestein-Jensen; sin embargo,
Tabla 2. Características de estudios complementarios y de LCR

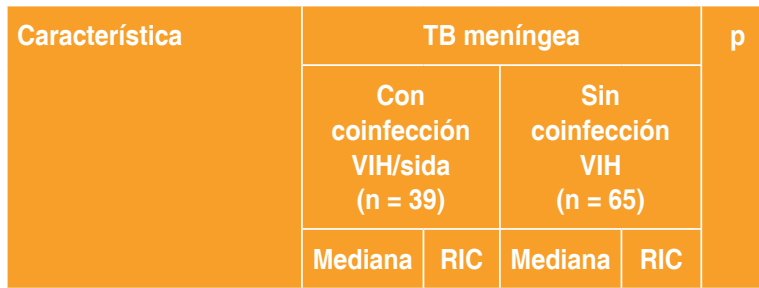

Citoquímica de LCR

al diagnóstico de

meningitis TB

Glucosa (mg/dL)

Proteínas $(\mathrm{mg} / \mathrm{dL})$

Células $/ \mathrm{mm}^{3}$

\begin{tabular}{|c|c|c|c|c|}
\hline 44 & 24 & 45 & 47 & 0.7 \\
81 & 159 & 115 & 1594 & 0.4 \\
37 & 125 & 96 & 298 & 0.03
\end{tabular}

ADA al ingreso (UI/L)

7

ADA seguimiento (UI/L)

\begin{tabular}{l|l}
7 & 13
\end{tabular}

7

$12 \quad 0.4$

$\mathrm{RIC}=$ rango intercuartilar, $\mathrm{LCR}$ = líquido cefalorraquídeo, $\mathrm{ADA}=$ adenosina

desaminasa.

solo se logró aislamiento en 21 pacientes (20\%), como se determinó en muestras biológicas: de líquido cefalorraquídeo en cinco casos (23\%), de secreción bronquial en seis $(29 \%)$ y de orina en 10 (48\%). La prueba de PCR GeneXpert en líquido cefalorraquídeo fue positiva en 15 pacientes (13\%). El estudio citoquímico de líquido cefalorraquídeo mostró características de inflamación y la celularidad fue mayor en el grupo con coinfección por VIH ( $p=0.03)$. No se identificaron diferencias significativas entre los grupos en cuanto a los niveles de la enzima adenosina desaminasa (ADA), ni al ingreso ni durante el seguimiento clínico (Tabla 2).

\section{Evaluación neurocognoscitiva}

En nuestra población se encontraron alteraciones neurocognoscitivas como pérdida significativa de la memoria a corto, mediano y largo plazo; así como de la capacidad del pensamiento, juicio, alteración de las habilidades ejecutivas, fluencia verbal e, incluso, alteraciones en el estado de ánimo, respecto al cual predominó la depresión leve según la evaluación con las pruebas MoCA y NEUROPSI. La evaluación con MoCA se realizó a los pacientes al ingreso hospitalario y durante el seguimiento en consulta externa, antes y después de los 12 meses posteriores al egreso. Al ingreso hospitalario, el promedio en los pacientes con coinfección por VIH/sida fue $23.4 \pm 5.7$, en el seguimiento antes de los 12 meses fue $24.3 \pm 4.4$ $(p=0.01)$ y después de los 12 meses de $26.6 \pm 0.5$ 
$(p=0.02)$; los pacientes sin coinfección por $\mathrm{VIH}$, al ingreso mostraron $24.1 \pm 5.3$, en el seguimiento antes de los 12 meses $23.7 \pm 5.6$ y $24 \pm 7.1$ después del año de seguimiento.

En 16 pacientes (41\%) con coinfección por $\mathrm{VIH} / \mathrm{sida}$ se observó alteración visuoespacial, de la identificación en seis (15.4\%), del lenguaje en 12 (30.8\%), de la abstracción en 12 (30.8\%), de la memoria en $20(51.3 \%)$ y de la orientación en 13 (33.3\%). En el grupo sin coinfección por VIH se observaron alteraciones en 23 pacientes (37.7\%), de la identificación en ocho (13.1\%), del lenguaje en $17(27.4 \%)$, de la abstracción en 16 (25.8\%), de la memoria en $30(47.3 \%)$ y de la orientación en 16 (25.8\%). No hubo diferencias estadísticamente significativas entre los grupos (Tabla 3).

La atención se encontró más frecuentemente alterada en pacientes con coinfección por $\mathrm{VIH} /$ sida (26 casos, $66.7 \%$ ), lo que se tradujo en relevancia estadísticamente significativa $(p=0.02)$

No hubo diferencias significativas en el grado de la severidad del deterioro entre los grupos; sin embargo, los pacientes en quienes al ingreso hospitalario las manifestaciones se iniciaron con alteraciones en el deterioro neurocognoscitivo fueron más propensos a desarrollar afección posterior permanente $(p=0.001)$.

\section{Tratamiento}

Todos los pacientes recibieron DOTBAL (rifampicina, pirazinamida, etambutol e isoniazida), piridoxina y dexametasona $0.3 \mathrm{mg} /$ día durante tres semanas, con posterior desescalamiento con prednisona oral durante su hospitalizaron. La fase intensiva del tratamiento antifimico consistió en 60 dosis, mientras que la fase de mantenimiento se prolongó por 15 a 18 meses, de acuerdo con los hallazgos de neuroimagen.

De 104 pacientes que recibieron el tratamiento de forma empírica, $70 \%$ presentó respuesta favorable (clínica y radiológica) al mismo.

La efectividad del tratamiento no mostró relevancia en la presencia de deterioro cognoscitivo ( $p=0.4)$, el grado de severidad del deterioro $(p=0.2)$ ni aparición de secuelas al egreso $(p=0.3)$.

\section{Discusión}

La TB meníngea continúa presentando una tasa de mortalidad elevada, por lo constituye un problema de salud pública alarmante. ${ }^{12}$ En esta investigación, muy
Tabla 3. Alteraciones, severidad y secuelas cognoscitivas de acuerdo con la prueba MoCA

\begin{tabular}{|c|c|c|c|c|c|}
\hline \multirow[t]{3}{*}{ Variable } & \multicolumn{4}{|c|}{ TB meníngea } & \multirow[t]{3}{*}{$\mathrm{p}$} \\
\hline & \multirow{2}{*}{\multicolumn{2}{|c|}{$\begin{array}{c}\begin{array}{c}\text { Con } \\
\text { coinfección } \\
\text { VIH/sida } \\
(n=39)\end{array} \\
\text { Media } \pm \text { DE }\end{array}$}} & \multirow{2}{*}{\multicolumn{2}{|c|}{$\begin{array}{c}\begin{array}{c}\text { Sin } \\
\text { coinfección } \\
\text { VIH } \\
(n=65)\end{array} \\
\text { Media } \pm \text { DE }\end{array}$}} & \\
\hline & & & & & \\
\hline \multirow[t]{2}{*}{$\begin{array}{l}\text { MoCA } \\
\text { Ingreso } \\
\text { Seguimiento }<12 \text { meses } \\
\text { Seguimiento }>12 \text { meses }\end{array}$} & \multicolumn{2}{|c|}{$\begin{array}{l}23.4 \pm 5.7 \\
24.3 \pm 4.4 \\
26.6 \pm 0.5\end{array}$} & \multicolumn{2}{|c|}{$\begin{array}{r}24.1 \pm 5.3 \\
23.7 \pm 5.6 \\
24 \pm 7.1\end{array}$} & \multirow[t]{2}{*}{$\begin{array}{l}0.9 \\
0.01 \\
0.02\end{array}$} \\
\hline & $\mathrm{n}$ & $\%$ & $n$ & $\%$ & \\
\hline $\begin{array}{l}\text { Alteración visuoespacial } \\
\text { Sí } \\
\text { No }\end{array}$ & $\begin{array}{l}16 \\
23\end{array}$ & $\begin{array}{l}41 \\
59\end{array}$ & $\begin{array}{l}23 \\
38\end{array}$ & $\begin{array}{l}37.7 \\
62.3\end{array}$ & 0.7 \\
\hline $\begin{array}{l}\text { Alteración identificación } \\
\text { Sí } \\
\text { No }\end{array}$ & $\begin{array}{c}6 \\
33\end{array}$ & $\begin{array}{l}15.4 \\
84.6\end{array}$ & $\begin{array}{c}8 \\
53\end{array}$ & $\begin{array}{l}13.1 \\
86.9\end{array}$ & 0.7 \\
\hline $\begin{array}{l}\text { Alteración atención } \\
\text { Sí } \\
\text { No }\end{array}$ & $\begin{array}{l}26 \\
13\end{array}$ & $\begin{array}{l}66.7 \\
33.3\end{array}$ & $\begin{array}{l}27 \\
35\end{array}$ & $\begin{array}{l}43.5 \\
56.5\end{array}$ & 0.02 \\
\hline $\begin{array}{l}\text { Alteración lenguaje } \\
\text { Sí } \\
\text { No }\end{array}$ & $\begin{array}{l}12 \\
27\end{array}$ & $\begin{array}{l}30.8 \\
69.2\end{array}$ & $\begin{array}{l}17 \\
45\end{array}$ & $\begin{array}{l}27.4 \\
72.6\end{array}$ & 0.7 \\
\hline $\begin{array}{l}\text { Alteración abstracción } \\
\text { Sí } \\
\text { No }\end{array}$ & $\begin{array}{l}12 \\
27\end{array}$ & $\begin{array}{c}0.8 \\
69.2\end{array}$ & $\begin{array}{l}16 \\
46\end{array}$ & $\begin{array}{l}25.8 \\
74.2\end{array}$ & 0.5 \\
\hline $\begin{array}{l}\text { Alteración memoria } \\
\text { Sí } \\
\text { No }\end{array}$ & $\begin{array}{l}20 \\
19\end{array}$ & $\begin{array}{l}51.3 \\
48.7\end{array}$ & $\begin{array}{l}30 \\
32\end{array}$ & $\begin{array}{l}47.3 \\
52.7\end{array}$ & 0.7 \\
\hline $\begin{array}{l}\text { Alteración orientación } \\
\text { Sí } \\
\text { No }\end{array}$ & $\begin{array}{l}13 \\
26\end{array}$ & $\begin{array}{l}33.3 \\
66.7\end{array}$ & $\begin{array}{l}16 \\
46\end{array}$ & $\begin{array}{c}5.8 \\
74.2\end{array}$ & 0.4 \\
\hline $\begin{array}{l}\text { Deterioro cognoscitivo } \\
\text { Sí } \\
\text { No }\end{array}$ & $\begin{array}{l}20 \\
19\end{array}$ & $\begin{array}{l}51.3 \\
48.7\end{array}$ & $\begin{array}{l}31 \\
34\end{array}$ & $\begin{array}{l}47.7 \\
52.3\end{array}$ & 0.7 \\
\hline $\begin{array}{l}\text { Secuelas cognoscitivas } \\
\text { Sí } \\
\text { No }\end{array}$ & $\begin{array}{l}21 \\
15\end{array}$ & $\begin{array}{l}58.3 \\
41.7\end{array}$ & $\begin{array}{l}38 \\
25\end{array}$ & $\begin{array}{l}60.3 \\
39.7\end{array}$ & 0.8 \\
\hline $\begin{array}{l}\text { Severidad } \\
\text { Moderada } \\
\text { Severa }\end{array}$ & $\begin{array}{c}11 \\
9\end{array}$ & $\begin{array}{l}55 \\
45\end{array}$ & $\begin{array}{l}13 \\
18\end{array}$ & $\begin{array}{l}41.9 \\
58.1\end{array}$ & 0.3 \\
\hline Población total & $\begin{array}{l}\text { Con } \\
\text { cog }\end{array}$ & $\begin{array}{l}\text { eterioro } \\
\text { scitivo }\end{array}$ & $\begin{array}{l}\operatorname{Sin} c \\
\operatorname{cog} r\end{array}$ & $\begin{array}{l}\text { terioro } \\
\text { scitivo }\end{array}$ & \\
\hline Secuelas al egreso & 36 & 76.6 & 23 & 44.2 & 0.001 \\
\hline
\end{tabular}

pocos pacientes presentaron TB pulmonar activa o como antecedente al momento del diagnóstico; al mismo tiempo, pocos pacientes tuvieron VIH como factor de riesgo, contrario a lo documentado en la 
literatura. ${ }^{13}$ Las manifestaciones clínicas y hallazgos en estudios de imagen fueron similares a los descritos en otras investigaciones, predominantemente cuadro de meningitis y colecciones. ${ }^{14,15}$

No se registró relevancia estadística en la presentación clínica, hallazgos de imagen ni características inflamatorias del líquido cefalorraquídeo en pacientes con o sin coinfección por VIH/sida.

Múltiples estudios en la literatura han demostrado que el deterioro cognitivo en las personas con VIH se encuentra estrechamente relacionado, en forma más relevante en personas que aún no cuentan con tratamiento. ${ }^{16} \mathrm{El}$ tratamiento antirretroviral eficaz retrasa exponencialmente el deterioro cognitivo. ${ }^{17}$

El deterioro cognoscitivo fue ligeramente mayor en los pacientes con VIH/sida (51.3\%) que en los pacientes sin coinfección por VIH (47.7\%); sin embargo, en los pacientes con $\mathrm{VIH} /$ sida predominó el deterioro moderado (55\%) y en los pacientes sin $\mathrm{VIH}$, el severo $(58.1 \%)$. Las secuelas cognoscitivas se presentaron en ambos grupos sin diferencias.

Aun cuando la TB del sistema nervioso central en pacientes con $\mathrm{VIH} /$ sida se asocia a mayor riesgo de deterioro cognoscitivo, no se demostró mayor grado de severidad ni de secuelas en estos pacientes. Durante el seguimiento se observó mejoría de las secuelas previamente definidas en ambos grupos (con y sin infección por VIH), con puntuaciones más altas en los pacientes con VIH/sida.

Las limitaciones de nuestra investigación estribaron en la imposibilidad para recabar los datos faltantes en los expedientes clínicos, al tratarse de un estudio retrospectivo; en la falta de seguimiento a largo plazo, puesto que no se pudo continuar con $100 \%$ de los pacientes debido a defunciones e inasistencia a la consulta de control en consulta externa; así como en el tamaño de la muestra. Por lo anterior, para mejorar los alcances de esta investigación, se debería realizar en una población mayor, por lo que un estudio multicéntrico nacional sería verdaderamente enriquecedor.

\section{Conclusiones}

En este estudio retrospectivo, analítico y transversal reportamos las alteraciones neurocognoscitivas en población mexicana con TB del sistema nervioso central con y sin coinfección por VIH. Las manifestaciones clínicas, de imagen y laboratorio correspondieron a las descritas en investigaciones nacionales e internacionales efectuadas en la última década. ${ }^{18-21}$ No hubo datos de relación estadísticamente significativa entre el deterioro cognoscitivo y la coinfección por VIH, probablemente por el tamaño pequeño de la muestra.

El deterioro cognoscitivo disminuye la calidad de vida en los pacientes. ${ }^{22}$ Las secuelas cognoscitivas mostraron mejoría en el seguimiento con el adecuado manejo y control terapéutico de los pacientes; por ende, es de suma importancia establecer el diagnóstico de forma oportuna e iniciar, en la medida de lo posible, el tratamiento efectivo y procurar la adherencia terapéutica para prevenir las complicaciones.

\section{Agradecimientos}

A todo el personal del laboratorio de infectología, con mención especial al doctor José Luis Soto Hernández.

\section{Financiamiento}

Los autores declaran que no recibieron financiamiento.

\section{Conflicto de intereses}

Los autores declaran que no tienen conflicto de intereses.

\section{Responsabilidades éticas}

Protección de personas y animales. Los autores declaran que para esta investigación no realizaron experimentos en seres humanos ni en animales.

Confidencialidad de los datos. Los autores declaran que siguieron los protocolos de su centro de trabajo sobre la publicación de datos de pacientes.

Derecho a la privacidad y consentimiento informado. Los autores declaran que en este artículo no aparecen datos de pacientes.

\section{Bibliografía}

1. World Health Organization [Internet]. Suiza: Global tuberculosis report 2019; 2020.

2. Chinyama J, Ngoma MS, Menon AJ, Hestad K, Heaton RK. The effect of pulmonary tuberculosis on neurocognitive function in HIV infected adult patients in Lusaka, Zambia. Med J Zambia. 2016;43:199-206.

3. Hunter RL. The pathogenesis of tuberculosis: the early infiltrate of post-primary (adult pulmonary) tuberculosis: a distinct disease entity. Front Immunol. 2018; 9:2108.

4. Behar SM, Divangahi M, Remold HG. Evasion of innate immunity by Mycobacterium tuberculosis: is death an exits strategy? Nat Rev Microbiol. 2010;8:668-674.

5. Chen HL, Lu CH, Chang CD, Chen PC, Chen MH, Hsu NW, et al. Structural deficits and cognitive impairment in tuberculous meningitis. BMC Infect Dis. 2015;15:279.

6. Gale SD, Erickson LD, Berrett A, Brown BL, Hedges DW. Infectious disease burden and cognitive function in young to middle-aged adults. Brain Behav Immun. 2016;52:161-168. 
7. Davis, AG, Nightingale S, Springer PE, Solomons R, Arenivas A, Wilkinson RJ, et al. Neurocognitive and functional impairment in adult and paediatric tuberculous meningitis. Wellcome Open Res. 2019;4:178.

8. Sanford AM. Mild cognitive impairment. Clin Geriatr Med. 2017;33:325-337.

9. Hestad KA, Chinyama J, Anitha MJ, Ngoma MS, McCutchan JA Franklin DR, et al. Cognitive impairment in Zambians with HIV infection and pulmonary tuberculosis. J Acquir Immune Defic Syndr. 2019;80:110 117.

10. Boletín Epidemiológico. Sistema Nacional de Vigilancia Epidemiológica Sistema Único de Información. Semana epidemiológica 36 [Internet] México: Secretaría de Salud; 2019.

11. Marais S, Thwaites G, Schoeman JF, Török ME, Misra UK, Prasad K, et al. Tuberculous meningitis: a uniform case definition for use in clinical research. Lancet Infect Dis. 2010;10:803-812.

12. Defunciones por tuberculosis todas formas 1990-2015 [Internet]. México: Centro Nacional de Programas Preventivos y Control de Enfermedades; 2015.

13. Wilkinson R, Rohlwink U, Misra U, van Crevel R, Dooley KE, Caws M, et al. Tuberculous meningitis. Nat Rev Neurol. 2017:13(10):581-598.

14. Cadena AM, Fortune SM, Flynn JL. Heterogeneity in tuberculosis. Nat Rev Immunol. 2017;17:691-702.
15. Pehlivanoglu F, Yasar KK, Sengoz G. Tuberculous meningitis in adults: a review of 160 cases. ScientificWorldJournal. 2012;2012:169028.

16. Devlin KN, Giovannetti T. Heterogeneity of neuropsychological impairment in HIV infection: contributions from mild cognitive impairment. Neuropsychol Rev. 2017;27:101-123.

17. Nightingale $S$, Winston $A$. Measuring and managing cognitive impairment in HIV. AIDS. 2017;31:S165-S172.

18. Furlow B. Tuberculosis: a review and update. Radiol Technol. 2010;82: 33-52.

19. Patkar D, Narang J, Yanamandala R, Lawande M, Shah GV. Central nervous system tuberculosis: pathophysiology and imaging findings. Neuroimaging Clin N Am. 2012:22:677-705.

20. Sáenz B, Hernandez-Pando R, Fragoso G, Bottasso O, Cárdenas G. The dual face of central nervous system tuberculosis: a new Janus Bifrons? Tuberculosis (Edinb). 2013:93:130-135

21. Schaller MA, Wicke F, Foerch C, Weidauer S. Central nervous system tuberculosis: etiology, clinical manifestations and neuroradiological features. Clin Neuroradiol. 2019;29:3-18.

22. Rubin LH, Maki PM. HIV, depression, and cognitive impairment in the era of effective antiretroviral therapy. Curr HIV/AIDS Rep. 2019;16: 82-95. 